\title{
Microstructure of the relaxed (001) Si surface
}

\author{
${ }^{1}$ A. E. Kiv, ${ }^{2}$ V. N. Soloviev, ${ }^{2}$ T. I. Maximova \\ ${ }^{1}$ Ben-Gurion university of the Negev, P.O.B. 653 Beer-Sheva \\ 84105 Israel, Tel./fax : +972-07-64614 60, E-mail : kiv@bgumail.bgu.ac.il \\ ${ }_{2}^{2}$ Pedagogical Institute, Krivoy Rog, 324086 Ukraine, E-mail: svn@kpi.dp.ua
}

\begin{abstract}
MD computer simulation with the Stillinger-Weber potential have been performed to study a microstructure of silicon surface layers and relaxation processes induced by low energy ion beams. New peculiarities of relaxed (001) silicon surface were discovered by using the improved calculation scheme for diamond-like structure simulation. It was established that the relaxed microstructure of clean (001) Si surface is characterized by dangling bonds in the first three near-surface layers and by non-hexagonal polygons. Besides, the dimer formation was observed not only in the first layer. New space configurations of dimers were found. Ascertained were some conditions which lead to the effect of radiation-stimulated relaxation of surface layers under the ion bombardment in the energy region of the threshold of elastic atomic displacements in silicon.
\end{abstract}

Keywords: silicon surface, molecular dynamics, radiation - stimulated processes.

Paper received 12.09.99; revised manuscript received 14.12.99; accepted for publication 14.03.00.

\section{Introduction}

The progress in understanding of $\mathrm{Si}(001)$ surface structure has been well described in a series of review papers and conference proceedings [1,2]. This surface has two dangling bonds per surface atom which move towards each other in pairs leading to $(2 \times 1)$ unit cell formation. Higher-order periodicities have also been observed as well as disordered configurations. Ab initio theoretical analysis of relaxation processes in semiconductor surface layers is limited by the great complexity of the phenomena involved, and thus forcing the use of simulation tools. The Molecular Dynamics (MD) method gives a useful insight into the problem. Many results (for example [3]) show that the MD method can provide a useful guide for exploring relaxation models for silicon surface, and what's more the simulation scheme may be optimized by simultaneous electronic calculations [4].

Conditions for relaxation of atomic configurations in semiconductor surface layers are fundamentally differed from those in the volume. Since surface layers contain non-saturated chemical bonds, the predominant processes of their rehybridization and rebuilding proceed under free relaxation of clean semiconductor surface. Experimental results show that oxide phase in clean silicon surface penetrates to several layers and reproduces the struc- ture of relaxed atomic configurations [5]. New algorithm of simulation of relaxation processes is necessary for description of real structure of relaxed silicon surface layers. Such algorithm, which allows to study a wide spectra of structural and electronic defects under relaxation of surface layers of silicon, was worked out and applied for MD simulation of $\mathrm{Si}(001)$ real surface structure.

A widespread technological process in microelectronics is radiation treatment of silicon surface. This process is used for surface cleaning and ion-assisted dry etching, but has the inherent drawback that the particle-surface interaction produces a distorted and disordered surface layers. It is of great interest to study destruction and relaxation processes induced by low-energy ion bombardment of silicon surface, in particular by self-ion implantation [6]. According to the ion energy, dose, dose rate, and the implantation temperature silicon surface layers can be damaged, amorphized and recrystallized [7]. The problem is to clarify conditions of the ion irradiation and to determine parameters of ion beams which may stimulate relaxation processes leading to the best surface characteristics.

In Sec. 2 we shall describe a scheme and details of calculations. Sec. 3, 4 and 5 contain the results of computer simulation of silicon surface structure, including ion-induced relaxation processes. 


\section{Calculation procedure}

A MD code is applied, which is suitable to investigate both surface features and bulk properties of silicon. The Stillinger-Weber potential was used [8]. The previous testing of the computer program has shown that with the time step $2.5 \cdot 10^{-15} \mathrm{sec}$ the relative error on the total energy is about $10^{-30} \%$, while the structural parameters are not affected.

A starting configuration was taken as a parallelepiped containing 864 atoms. It were 12 layers with 72 atoms in each one. Periodic boundary conditions were applied in two dimensions. Relaxation processes were studied at the temperatures 290 and $1000 \mathrm{~K}$.

By analogy to [9], the model is based on the assumption that each atom in the relaxation process may change its neighboring atoms and create chemical bonds with new four nearest atoms. So the relaxation is accompanied by rebuilding chemical bonds and their re-hybridization. Such procedure leads to arising of non-hexagonal polygons and dangling bonds.

The Table 1 illustrates a situation which leads to formation of dangling bonds.

Table 1. Illustration of dangling bonds formation

\begin{tabular}{c|c|c|c|c|c}
\hline \hline \multicolumn{2}{l}{ The central atom } & \multicolumn{4}{c}{ The nearest neighboring atoms } \\
\hline & 1 & 2 & 3 & 4 & 5 \\
\hline a) & 2 & 1 & 6 & 7 & 8 \\
\hline b) & 2 & 9 & 6 & 7 & 8 \\
\hline \hline
\end{tabular}

For example, let us suggest that the atom 1 is bonded with atoms 2, 3, 4 and 5. In the process of relaxation, the following situations may occur:

a) atom 2 may have the atom 1 as one of its neighboring atoms, b) the nearest neighbors of atom 2 may not include the atom 1 . Then in the case b) one bond of the atom 1 appears unsaturated. In the last situation one dangling bond arises in the node with atom 1 . An analogous situation in other nodes may lead to formation of two and three dangling bonds and also to formation of interstitial atoms. Computer programs allowed us to obtain radial distribution functions (RDF) and angle distribution functions (ADF) for angles between chemical bonds.

Further, the influence of bombardment of silicon surface by low energy neutral particles on relaxation processes was studied. The energies of bombarding particles were $10,20,30,40$ and $50 \mathrm{eV}$. In simulation procedure one impulse corresponded to $2 \cdot 10^{12}$ particles $/ \mathrm{cm}^{2} \cdot \mathrm{s}$.

\section{Quasi-disordered phase}

The results of computer simulation of Si surface relaxation processes have shown that a quasi-disordered phase (QDP) arises as a result of free silicon surface relaxation. Only four near-surface layers form the QDP. The model has emphasized that in particular the microstructural constituents in silicon surface layers are nodes with one or more dangling bonds. One can see that the RDF and ADF are similar to those for a-Si (Fig. 1). For the ADF the main difference between silicon surface and aSi structure is situated in the region of $60^{\circ}$. As a result of relaxation non-hexagonal polygons and dangling bonds were discovered in silicon near-surface layers. The fifth layer does not differ practically from more deep layers.

Each of four layers which form the QDF has his specific structural characteristics. The third layer has less structural and electronic defects in comparison with other layers of QDP. Calculations have shown that this layer almost has not dangling bonds. But at the same time the third layer is the most distorted one. The Table 2 gives displacements upwards and downwards of atoms belonging to different surface layers. As we see, atoms of the third layer are characterized by the largest displacements upwards relatively to the normal position of the corresponding crystallographic plane (1.8712 $\AA$ ). Only for the first layer atom displacements are larger $(1.1883 \AA)$, but it is naturally. In the Table 3 we can also see that the third layer on the whole has the largest displacements to the surface. As a result of relaxation the distance between the third and the fourth layers became $1.586 \AA$ and between the third and the second layers became $1.082 \AA$. Analysis of microstructure of near-surface layers of silicon indicates that the third layer plays a special role in stabilization of QDP. It may be concluded that this layer is a transitional one between the crystal volume and the surface layers.

\section{Structure of dimers in relaxed $\mathrm{Si}(001)$ sur- face}

Because of the structure of relaxed silicon surface layers contains dangling bonds, the conditions arise for formation of dimers not only in the first layer. In near surface layers new space configurations of dimers were obtained.

Table 2. Displacements of atoms in surface layers relatively to their position before relaxation

\begin{tabular}{c|l|l|l|l}
\hline \hline & \multicolumn{3}{|c}{ Displacement (̊) } \\
\hline Layers & \multicolumn{2}{|c}{$U p$} & \multicolumn{2}{c}{ Down } \\
\hline & Min & Max & Min & Max \\
\hline 1 & 0.2131 & 1.1883 & 0.1010 & 2.7905 \\
\hline 2 & 0.0040 & 1.4798 & 0.0461 & 1.6952 \\
\hline 3 & 0.1782 & 1.8712 & 0.0031 & 1.2565 \\
\hline 4 & 0.0046 & 0.7731 & 0.0041 & 0.6137 \\
\hline 5 & 0.0106 & 0.2653 & 0.0061 & 0.3874 \\
\hline \hline
\end{tabular}

Table 3. The mean distances between $j$ and $j+1$ surface layers after relaxation

\begin{tabular}{l|l|l|l|l|l|l|l}
\hline \hline \multicolumn{7}{c}{ Distances between $j$ and $j+$ layers $(\AA)$} \\
\hline 1,2 & 2,3 & 3,4 & 4,5 & 5,6 & 6,7 & 7,8 & 8,9 \\
\hline 0.535 & 1.082 & 1.586 & 1.328 & 1.345 & 1.347 & 1.346 & 1.346 \\
\hline \hline
\end{tabular}




\section{A.E. Kiv et al.: Microstructure of the relaxed (001) Si surface.}

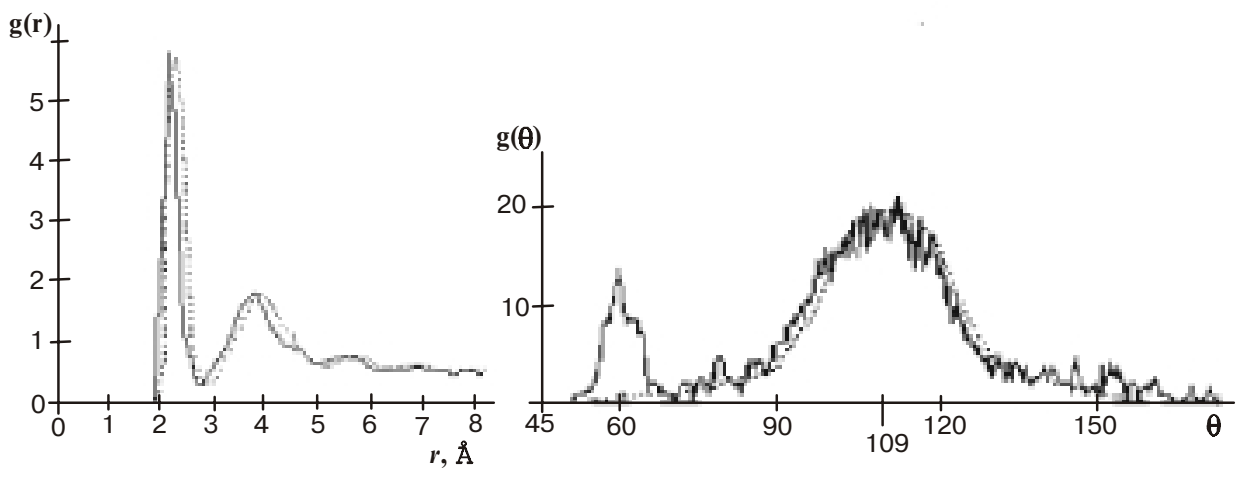

Fig. 1. Radial distribution functions (left) and angle distribution functions for angles between chemical bonds (right) in relaxed nearsurface layers of silicon. Dotted lines correspond to a-Si.

As a rule [10] dimers are investigated by moving only atoms of the first layer by equal and opposite distances along the given direction. By using the simulation technique which gives a full relaxation of the system and rehybridization of chemical bonds we obtained new dimer configurations besides those which are described in known works (for example $[10,11]$ ).We have discovered dimers not only in the first layer and have observed their specific space orientations as well as size dispersion (Fig. 2 ).

Configurations of dimers correlate with the number and the type of structural and electronic defects in each surface layer. The density of dimers in the first layer is by the order of magnitude larger than in the second layer and become especially small in the third and in the fourth layers.

It was established that in the second and in the third layers there is a large part of dimers which dispose under large angles of inclination to the (001) plane. In the forth layer there are few dimers, and they almost all dispose in the (001) plane.

In the first layer the most of dimers is situated in the vicinity of direction $\vec{d}_{0}\left(\vec{d}_{0}\right.$ shows the direction of ideal dimers [3]). In the forth layer dimers dispose perpendicular to $\vec{d}_{0}$.

The mean size of dimers for each layer is shown in the Table 4. For the first layer we obtained the dispersion of dimer sizes in the interval $0.217-0.250 \mathrm{~nm}$. Experimental measurements give $0.220-0.247 \mathrm{~nm}$ [15].
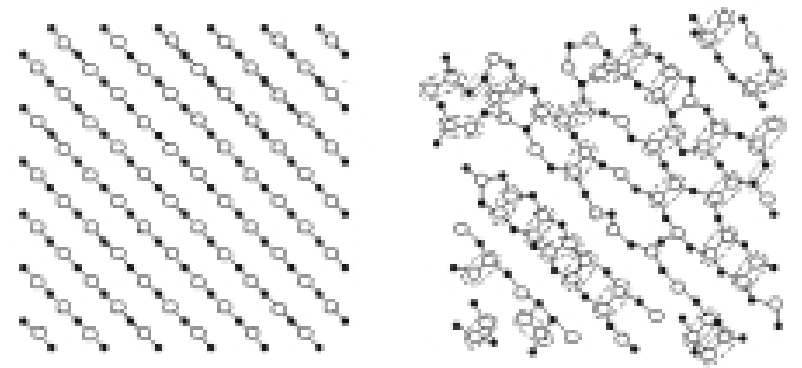

Fig. 2. The structure of the first silicon layer before relaxation (left) and formation of dimers after relaxation (right). Dimers are shown by the dotted lines.
Table 4. The mean sizes of dimers in relaxed silicon surface layers

\begin{tabular}{cc}
\hline \hline Number of the layer & The mean dimer size, $\mathrm{nm}$ \\
\hline 1 & 0.232 \\
\hline 2 & 0.229 \\
\hline 3 & 0.228 \\
\hline 4 & 0.236 \\
\hline \hline
\end{tabular}

The atom configurations of QDP sufficiently differ from initial unrelaxed near surface structure. Therefore speaking about some layer in relaxed QDP we imply the corresponding ideal layer before relaxation.

\section{Radiation treatment of silicon surface}

We investigated radiation effects in silicon surface layers caused by low-energy ion bombardment. The results were obtained for the transfered energies near the threshold $E_{d}$ for atom displacements in silicon lattice under the elastic collisions [12]. For monoenergetic mono-isotopic ion bombardment the induced structural changes are depended on the bombardment angle. Therefore, we have compared the results for different energies of ion beams at the same bombardment angles, ion doses and dose rates.

There are structural processes in this energy region of $E_{d}$ which are known as "grasshopper effect" in diamond-like lattices [13]. It was shown in [13] that the displacement of atoms in silicon lattice in the (111) direction under the transfered energy $E \cong E_{d}$ leads to arising of metastable atom configuration with unbroken chemical bonds. The relaxation of such configuration provides its displacement on the whole and stimulate migration of defects as well as a recovery of disordered regions.

We obtained a specific energy dependence of radiation-stimulated relaxation processes. It was just established that the ion irradiation of silicon surface in vicinity of $E_{d}$ leads to extreme values of structural characteristics of near surface layers. In Fig. 3a one can see the energy dependence of parameter $\mathrm{D} E$ that characterizes the level of relaxation. This parameter gives a relative 


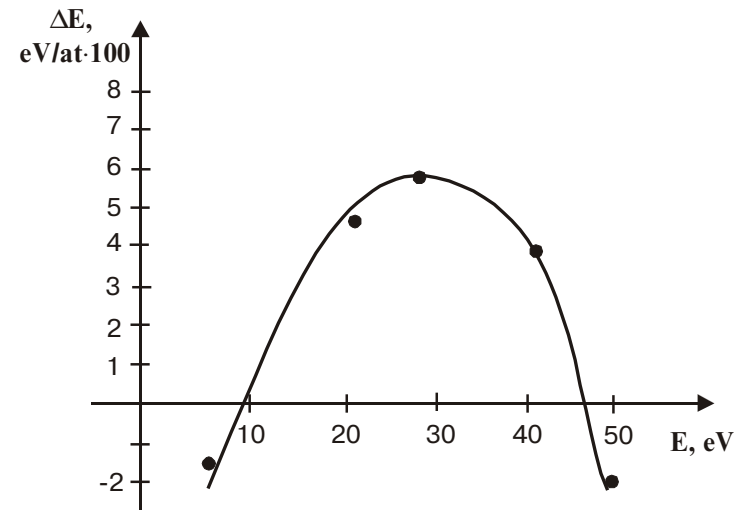

a)

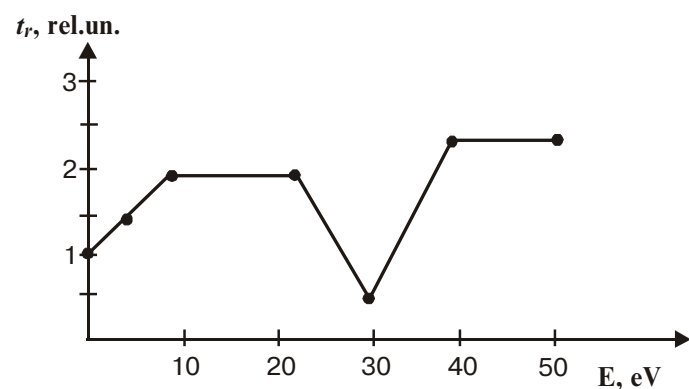

b)

Fig. 3. Energy dependences (a) of the level of relaxation and (b) of the relaxation time (explanation is in the text)

difference between the full energies for the initial state of the system and the state after final relaxation. The largest value of $\mathrm{D} E$ corresponds to the transfered energy $E \sim E_{d}$. The Fig. 3b shows the energy dependence of the relaxation time. For this parameter the optimum energy also lies in the vicinity of $E_{d}$.

Thus, it was established that ion bombardment of silicon surface in the energy region of the threshold of elastic displacement of atoms may allow to improve structural characteristics of surface layers and decrease the relaxation time.

\section{Conclusions}

MD simulation of atomic configurations in silicon surface layers with using the Stillinger-Weber potential allowed to study the real structure of free surface of silicon crystal at super high vacuum. The relaxation of the calculated cluster on the whole was studied. Besides, a special algorithm was used which included rebuilding of chemical bonds in to the relaxation process. As a result of such calculation procedure it was established that silicon surface layers under conditions of super high vacuum and full relaxation form QDP which is characterized by dangling bonds and non-hexagonal polygons. The QDP determines the structure of oxide phase which is formed on the natural silicon surface. These oxide films have a disordered structure and includes three-four surface layers [14].

Because of existing unsaturated bonds in near surface silicon layers, dimers arise not only in the first layer. Dimers in different surface layers have some peculiarities: their space orientations change from layer to layer.

Computer simulation of radiation treatment of silicon surface was performed for the case of bombardment by neutral ions with energies in the region of threshold of defect formation under elastic mechanisms. Energy dependences of radiation induced processes were obtained, which show a possibility to improve the real structure of silicon surface and to accelerate the long term surface relaxation in microelectronic technology.

\section{References}

1. G. P. Srivastova, Rep. Prog. Phys, 60, N5 pp. 561-613 (1997).

2. F. Bechstedt, R. Enderline, Semiconductor Surfaces and Interfaces (Their Atomic and Electronic Structures), Akademie-Verlag, Berlin (1988).

3. F. F. Abraham, I. P. Batra, Surf. Sci. 163, L752-L758 (1985)

4. Z. M. Khakimov. Computer Mater. Sci.3 (1), pp. 94-108 (1994).

5. I. P. Lisovskyy, V.G. Litovchenko, W. Fussel, A. E. Kiv, Intern. Conf. on POLYSE 2000.

6. P. X. Zhang, I. V. Mitchell, B. Y. Tong et al, Phys. Rev, B 50 (23) pp. 17080-17084, (1994).

7. L. J. Huang, W. M. Lau, H. T. Tang et al, Phys. Rev, B 50 (24) pp.18453-18468, (1994).

8. F. H. Stillinger, T. A .Weber, Phys .Rev, B 31 (2) pp. 52625267, (1984)

9. P.W. Jacobs, A.E. Kiv, R.M. Balabay et al, Computer Modelling\&New Technologies, 3 (1), p.15-21, (1998)

10. Farid F. Abraham, Inder P. Batra, Surface Sci. Lett. 163 (2), L752, (1985)

11. J. Dabrovski, M. Scheffler, Appl. Surf. Sci. 56-58 (1), pp. 1522, (1992).

12. V. S. Vavilov, A. E. Kiv, O. R. Niyazova, Mechanisms of formation and migration of defects in semiconductors, Nauka, M., 1981.

13. A. E. Kiv, V. N. Soloviev, Phys. Stat. Sol. (b), 94, pp. k91k95, (1979), (in Russian).

14. Ivor Brodie and Julius J. Muray, The Physics of Microfabrication, Chap 5, Plenum Press, New York and London, (1982)

15. L.S.O. Johansson, R.I.G. Uhrberg, P. Martensson and G. V. Hansson, Phys. Rev, B42 (1), pp. 1305-1310, (1990) 